\title{
Monocouches de billes de latex ou de silice : utilisation pour la calibration et pour le contrôle des pointes en AFM
}

\author{
M.-C. Boisset et C. Frétigny \\ ESPCI/LPQ, CNRS URA 1428, 10 rue Vauquelin, 75231 Paris Cedex 05, France
}

(Reçu le 20 octobre 1993; accepté le 20 décembre 1993)

\begin{abstract}
Résumé. - Nous proposons d'utiliser des billes de latex ou de silice déposées sur mica ou sur silicium pour contrôler la calibration des têtes de déplacement en AFM. Une méthode statistique de mesure des hauteurs par histogramme est donnée. De plus, il est montré que les mêmes échantillons peuvent servir à caractériser la qualité de la pointe.
\end{abstract}

\begin{abstract}
We propose the use of latex or silica balls deposited on mica or silicon to control the calibration of the scanners in AFM. A statistical method for the height measurement, based on an histogram analysis is given. Moreover, it is shown that the same samples can be used for tip characterisation.
\end{abstract}

\section{Introduction.}

La détermination précise de la taille des structures en microscopie de proximité est conditionnée par la calibration des déplacements du tube piézo-électrique. Pour beaucoup d'appareils commerciaux, ce sont les mêmes tubes qui sont utilisés en AFM et en STM; la calibration peut donc servir aux deux techniques. Elle doit être révisée périodiquement et il est donc important de disposer d'échantillons de référence faciles à imager et présentant des tailles caractéristiques. De plus, à cause de la non-linéarité de la réponse de la céramique piézo- électrique [1], il semble important d'utiliser des standards correspondants à différentes gammes de dimension $(1 \mathrm{~nm}$ à $5 \mu \mathrm{m})$. Pour de très petites hauteurs $(<10 \mathrm{~nm})$, des essais sur des particules d'argiles ont montré que l'erreur commise avec une tête calibrée à plusieurs centaines de nanomètres pouvaient atteindre un facteur 2. Ces particules peuvent donc servir à calibrer dans ce domaine [2]. Pour des hauteurs plus importantes ( $50 \mathrm{~nm}-1 \mu \mathrm{m})$, il a été proposé d'utiliser des échantillons constitués de billes de latex déposées sur mica [3].

D'autre part, une difficulté expérimentale importante, lors de l'étude AFM d'échantillons inconnus, est la reconnaissance des artefacts liés à la forme de la pointe (pointe émoussée, dissymétrique, salie, effets d'encombrement...). Un contrôle a posteriori est parfois nécessaire. 
Un échantillon dont les caractéristiques géométriques sont bien définies, comme par exemple une surface de InP comprenant des objets très fins et hauts [4] peut servir de modèle pour caractériser la pointe.

Dans cette communication, nous montrons comment des billes de silice ou de latex peuvent facilement servir à la fois comme standard de calibration et comme test de la qualité de la pointe.

\section{Les échantillons.}

On trouve dans le commerce des suspensions colloïdales de billes de latex ou de silice. Leur diamètre, très uniforme, se situe dans la gamme de $50 \mathrm{~nm}$ à plusieurs microns. La dispersion des tailles est de l'ordre de $1 \%$. Ces matériaux sont déjà utilisés comme standard de calibration en microscopie électronique. Dans le cas de l'AFM, la préparation consiste à déposer sur un substrat de mica fraîchement clivé ou de silicium, une goutte de suspension convenablement diluée et séchée à l'air. Pour une suspension initiale de billes de diamètre $\Phi$ à $\delta \%$ en masse, on peut aisément montrer que pour obtenir une fraction $\alpha$ de monocouche, à partir d'une goutte de volume $V$ séchée sur une suface $S$, il faut diluer d'un facteur $\delta^{\prime}$ :

$$
\delta^{\prime}=\frac{\pi}{3 \sqrt{3}} \frac{\alpha}{\delta} \frac{\rho_{\mathrm{B}}}{\rho_{\mathrm{E}}} \frac{S \Phi}{V}
$$

où $\rho_{\mathrm{B}}$ et $\rho_{\mathrm{E}}$ sont les densités des billes et de l'eau. Si $\delta=10 \%$ par exemple, et pour un facteur de dilution de $6.4 \times 10^{-3}$ sur une surface de $1 \mathrm{~cm}^{2}$ on obtient la relation :

$$
V=\frac{\alpha}{10} \Phi
$$

où $V$ est exprimé en $\mu \mathrm{l}$ et $\Phi$ en nm . $\alpha$ de l'ordre de $3 / 4$ semble être une bonne valeur pour pouvoir observer à la fois le substrat et des îlots de billes de taille raisonnable sans être gêné par une croissance tridimensionnelle. On obtient, le plus souvent, une surface constituée d'îlots denses séparés par des régions de substrat nu. Cependant,après sèchage, la densité de billes il est possible de trouver des zones de densité convenable en choisissant le point d'analyse. En pratique, les fonctions chimiques présentes à la surface des sphères déterminent la coalescence des îlots en contrôlant les interactions latérales et verticales des sphères.

La figure 1 présente une image typique obtenue avec des billes de $300 \mathrm{~nm}$ de diamètre. Les grands îlots servent à la calibration $x, y$ des tubes ainsi qu'au contrôle de la pointe, tandis que les plus petits permettent de calibrer les déplacements verticaux.

Pour obtenir des résultats corrects, il est important d'utiliser soit des latex dont la température de transition vitreuse est assez élevée, soit des billes de silice de façon à éviter les effets de "matière molle"qui rendent les hauteurs mesurées et les contrastes dépendants de la vitesse de balayage et de la force appliquée $[5,6]$.

\section{Calibration.}

3.1 DireCtion $x, y$. - L'arrangement compact des sphères permet une mesure statistique de leur taille apparente, par exemple, en utilisant l'autocorrélation de l'image comme le montre la figure 2. Les valeurs moyennes des distances séparant les centres des billes sont ainsi obtenues. Le nombre de billes sur l'image ne doit pas être trop faible pour que la moyenne ait un sens. Il ne doit pas être non plus trop important pour que la discrétisation de l'image n'introduise pas une incertitude trop importante - une précision relative du diamètre de $1 \%$ est obtenue avec 25 


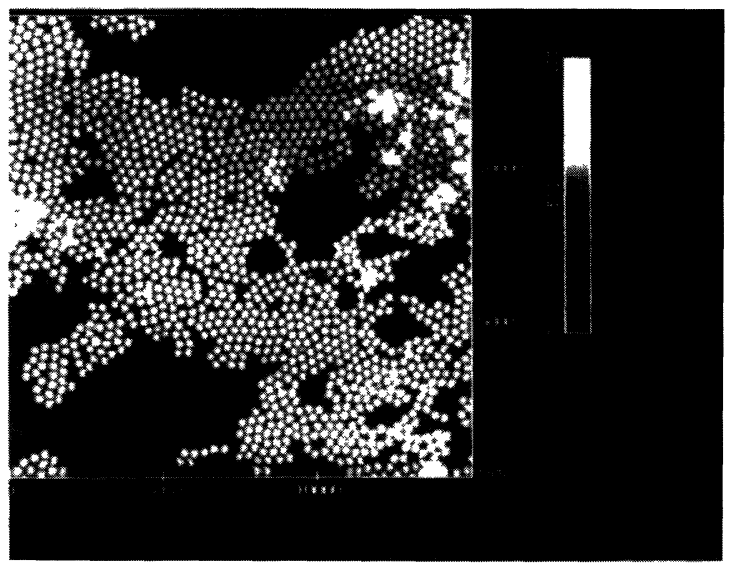

Fig. 1. - Image typique d'un dépôt de billes de latex sur un substrat de mica ou de silice. [Typical image of latex balls deposited on a mica or silicon substrate.]

billes pour une image $512 \times 512$. Il est clair que des échantillons micro-gravés pour l'électronique fournissent des standards de qualité comparable dans la direction $x$, Lavantage des billes est qu'elles permettent en plus la calibration dans la direction $z$.
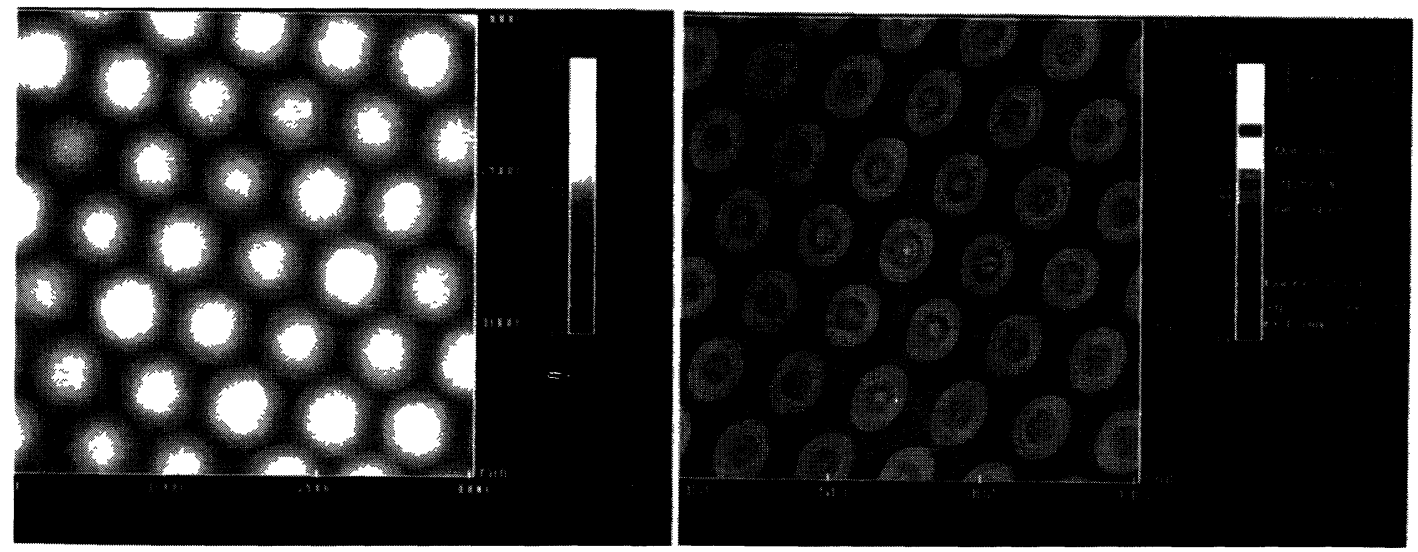

Fig. 2. - Région compacte d'un îlot de billes de silice et autocorrélation de l'image. Le diamètre moyen expérimental est donné par les premiers maxima autour du centre de l'autocorrélation.

[Compact zone of a silica balls island and autocorrelation of the image. The mean diameter is given by the first maxima neighbouring the center of the autocorrelation data.]

3.2 DireCtion $z$. - Pour déterminer les diamètres verticaux moyens apparents des sphères, il est nécessaire d'effectuer des images de régions dont certaines sont du substrat nu. Pour effectuer une moyenne cohérente sur les hauteurs mesurées, le substrat doit être impérativement plat. En effet, des "surfaces de base"souvent liées à des dérives électroniques ou thermiques rendent 
difficile l'analyse des hauteurs sur de grands îlots. Il est donc préférable de travailler sur de petits domaines entourés de substrat. La surface de base du substrat est définie sur les bords de l'image puis retirée en l'extrapolant sous les billes. Ce type de traitement, très simple, est disponible sur les appareils commerciaux. Il faut noter que l'image enregistrée doit être exempte de tout prétraitement automatique, comme le retrait de droite moyenne sur chaque ligne de balayage souvent proposé à l'acquisition. Il faut aussi prendre garde à utiliser au maximum le codage numérique des hauteurs disponible. Une image intéressante pour ce type de calibration est présentée en figure 3 .

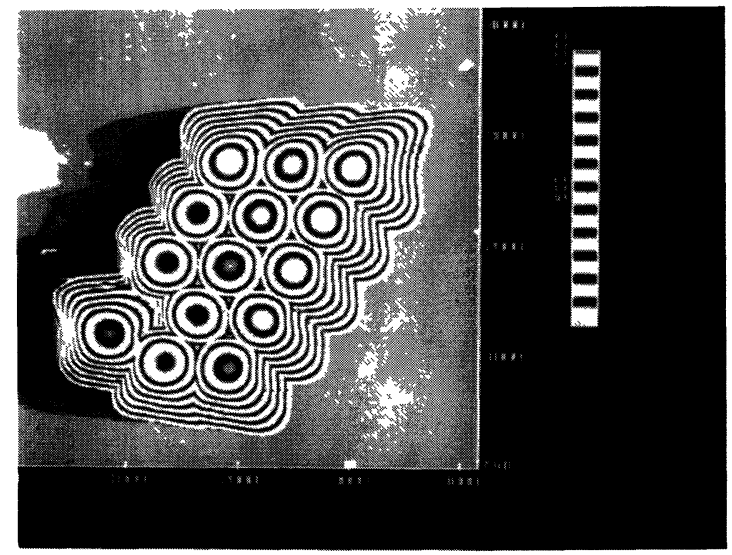

Fig. 3. - Petit îlot de billes. Une surface de base a été retirée, le substrat apparaît plat sur une bonne partie de l'image. La forme des billes en bord d'îlot reflète l'encombrement "pyramidal" de la pointe qui a été utilisée pour acquérir l'image.

[Small balls island. A base surface has been substracted, the substrate appears to be flat over a large area of the image. The shape of the balls at the edge of the island is characteristic of the tip's pyramidal shape used to obtain this image.]

Plutôt que d'effectuer de multiples mesures de hauteur, souvent fastidieuses puisqu'il faut déterminer les sommets de chaque bille, nous proposons une analyse fondée sur l'histogramme des hauteurs de l'image. Pour une surface dont l'équation est $z=f(x, y)$, l'expression de l'histogramme $H(z)$ est donnée par :

$$
H(z)=\iint \mathrm{d} x \mathrm{~d} y \delta(z-f(x, y))
$$

soit

$$
H(z)=\oint_{C(z)} \frac{\mathrm{d} s}{|\nabla f(x, y)|}
$$

où $C(z)$ est la courbe intersection entre la surface et le plan de hauteur $z$.

Dans la cas de sphères parfaites isolées, on peut montrer que l'histogramme a une forme triangulaire. La conséquence d'une légère dispersion des diamètres des billes peut être décrite par une convolution de l'histogramme avec une courbe en cloche. Le substrat, quant à lui, correspond à un pic théoriquement infiniment mince et très haut. Par rapport à ce cas idéal, le substrat n'est 
pas un plan horizontal, mais peut être incliné ou présenter une courbure qui provient des restes non corrigés après le retrait de la surface de base. On peut aussi, en première approximation, rendre compte de cet effet par une convolution par une courbe en cloche de l'ensemble de l'histogramme. La distance entre le point d'inflexion et le sommet du pic du substrat représente alors le diamètre moyen des billes. Les distorsions de l'image imposées par l'encombrement de la pointe ne modifient pas le sommet des billes qui reste sphérique, et n'affectent donc pas la partie de l'histogramme correspondante. La hauteur correspondant au point d'inflexion est invariante et la mesure du diamètre moyen reste valable. Ceci reste vrai tant que les élargissements sont faibles devant le diamètre des billes, ce qui est en général réalisé dans la pratique. La figure 4 montre l'histogramme correspondant à la zone entourée de la figure 3.

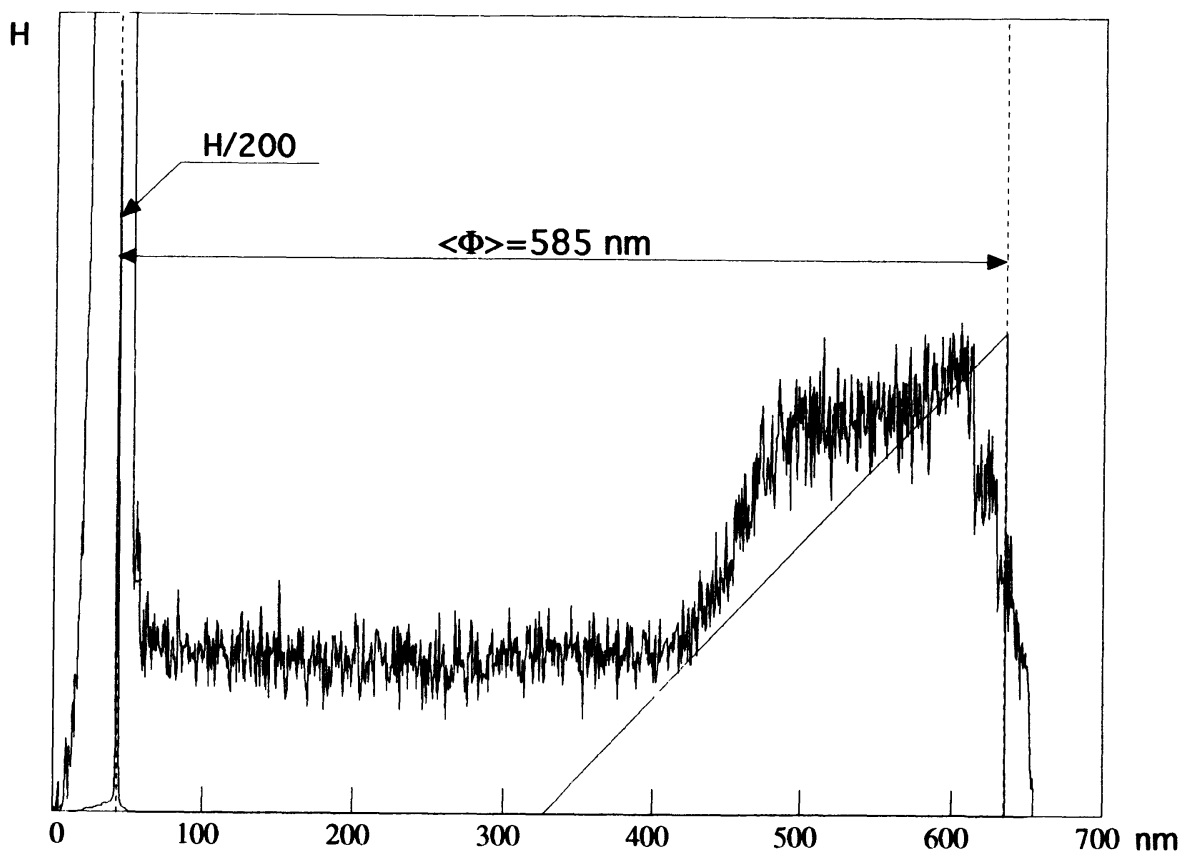

Fig. 4. - Histogramme des hauteurs calculé sur la figure 3. La mesure de la hauteur moyenne des sphères se fait entre le pic du substrat et le point d'inflexion de la courbe correspondant aux billes. Est aussi représentée une forme triangulaire correspondant à l'histogramme d'une bille isolée. Les écarts entre les deux courbes proviennent de la dispersion des diamètres, de la disposition en îlots et de la forme de la pointe.

[Height histogram calculated from Fig. 3. The average height of the balls is given by the distance between the substrate peak and the point of inflexion of the curve corresponding to the balls. A triangular shape corresponding to the histogram of an isolated ball is also presented. The difference between the two curves arises from the dispersion of the diameters, the island's arrangement and from the tip shape.]

\section{Forme de pointe.}

Les effets de forme géométrique de pointe peuvent être très importants, en particulier lorsque l'on image des surfaces présentant une forte rugosité aux hautes fréquences spatiales [7,8]. Par exemple, l'encombrement de la pointe empêche la visualisation de détails sur des fronts raides ou 
l'analyse de détails au fond de tranchées étroites. A plus petite échelle, le rayon de courbure du bout élargit les détails de petite dimension.

D'un autre côté, les pointes sont de qualité très diverses dans un même wafer [8]. Certaines présentent des défauts qui modifient l'aspect de l'image sans que les effets produits soient directement repérables. Des formes géométriques simples comme la sphère de silice ou de latex permettent, dans une certaine mesure, de caractériser la forme de la pointe.

Sur la figure 3, obtenue avec une pointe pyramidale, la forme générale des billes en bord d'îlot traduit l'effet d'encombrement de la pointe puisque les faces de la pyramide sont clairement visibles. Les angles des faces par rapport au plan correspondent bien à ceux de la pointe inclinée d'environ $10^{\circ}$.

En plus de cet effet d'encombrement, on peut chercher à mettre en évidence un éventuel effet du rayon de courbure du bout de pointe en étudiant une section suivant une rangée de billes d'un arrangement compact de sphères [5]: d'après la mesure du relief relatif observé, $\delta / \Phi$, on peut déduire que le relief est limité soit par l'angle d'ouverture de la pointe (Fig. 5a) soit par le rayon effectif du bout de pointe (Fig. 5b).

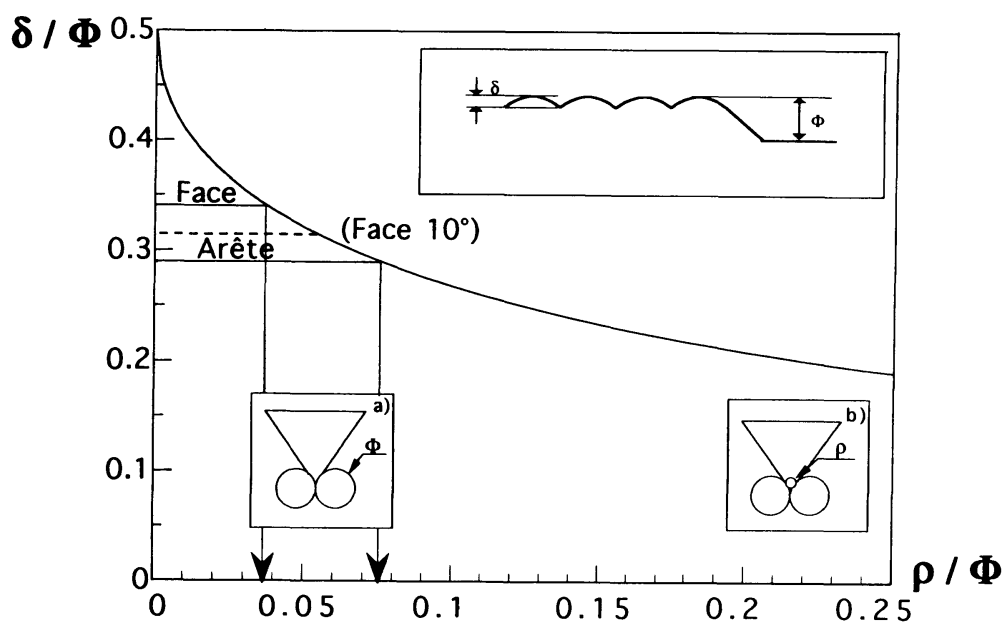

Fig. 5. - Position relatives de la pointe et des sphères dans le cas ou la descente est limitée par a) l'encombrement de la pointe ou b) par le bout de la pointe. La courbe présente le relief relatif au diamètre des billes dans les deux cas.

[Relative position of the tip and the spheres in the case where its position is imposed by a) the tip overall shape or b) by the tip end. The curve represents the relative corrugation in both cases.]

Dans le premier cas, on peut montrer que $\delta / \Phi$ est indépendant du diamètre des sphères. Sa valeur est caractéristique de la géométrie de la pointe. Si on étudie par exemple une rangée orientée suivant les faces de la pyramide ( $x$ ou $y$ ), sachant que les faces forment entre elles un angle de $70^{\circ}$, et que la pointe est inclinée de $10^{\circ}$ sur la verticale, un calcul simple montre que le relief relatif est :

$$
\frac{\delta}{\Phi}=0,32
$$

qui est à comparer avec la valeur de 0,5 qui serait obtenue pour une pointe infiniment fine. 
Dans le cas où la descente de la pointe est limitée par son rayon effectif de bout, le relief relatif dépend du diamètre des sphères comme représenté sur la figure 5 [5].

La moyenne des mesures du rapport $\delta / \Phi$ sur plusieurs régions, comparée aux valeurs de la courbe, permet alors de trouver le facteur limitant le relief. Dans le cas où c'est le bout de pointe qui limite, il est possible d'avoir une estimation de son rayon effectif. Dans le cas où c'est l'encombrement qui limite, on peut donner une borne supérieure à ce rayon. Avec les caractéristiques géométriques données plus haut, on peut montrer que pour mettre en évidence des rayons de courbure de l'ordre de $r$, il faut utiliser des billes de diamètre inférieur à environ $20 r$. Pour des billes de $100 \mathrm{~nm}$, le rayon effectif minimal que l'on peut mettre en évidence est en principe de $5 \mathrm{~nm}$. Dans ce cas, cependant, la détermination du relief est difficile à obtenir avec une bonne précision.

\section{Conclusion.}

Nous avons montré comment des échantillons constitués de billes de latex ou de silice déposées sur mica ou silicium pouvaient constituer de bonnes références pour la calibration des déplacements du tube piézo-électrique, mais aussi servir de test pour la qualité de la pointe. Il paraîtrait intéressant de pouvoir disposer de billes de diamètre encore inférieur pour élargir la gamme des contrôles.

\section{Remerciements.}

Nous avons le plaisir de remercier Jean-Pierre Aimé pour les nombreuses et fructueuses discussions que nous avons eues.

\section{Bibliographie}

[1] Hues S.-M., Draper C.-F., Lee K.-P., Colton R.-J., à paraître.

[2] Blum A.-E., Eberl D.-D., Water-Rocks Interaction Kharaka and Maest éd. (1992) Balkema, Rotterdam.

[3] Li Y., Lindsay S.M., Rev. Sci. Instrum. 62 (1991) 2630.

[4] Montelius L., Tegenfeldt J.-O., Appl. Phys. Lett. 62 (1993) 21.

[5] Odin C., Aimé J.-P., Elkaakour Z., Bouhacina T., à paraître.

[6] Granier V., Sartre A., Joanicot M., Euradh'92, Karlsruhe, (Septembre 1992).

[7] Grutter , Zimmermann-Edling W., Brodbeck D., Appl. Phys. Lett. 60 (1992) 2741.

[8] Flanders D.C., Efremow N.N., J. Vac. Sci. Techn. B1 (1983) 4.

Ohmi T., Aoyama S., Appl. Phys. Lett. 61 (1993) 2479. 